\title{
Statistical Convergence of Sequences of Functions in Intuitionistic Fuzzy Normed Spaces
}

\author{
Vatan Karakaya, ${ }^{1}$ Necip Şimşek, ${ }^{2}$ \\ Müzeyyen Ertürk, ${ }^{3}$ and Faik Gürsoy ${ }^{3}$ \\ ${ }^{1}$ Department of Mathematical Engineering, Yildiz Technical University, Davutpasa Campus, Esenler, \\ 34210 Istanbul, Turkey \\ ${ }^{2}$ Department of Mathematics, Istanbul Ticaret University, Uskudar, 34672 Istanbul, Turkey \\ ${ }^{3}$ Department of Mathematics, Yildiz Technical University, Davutpasa Campus, Esenler, \\ 34220 Istanbul, Turkey
}

Correspondence should be addressed to Vatan Karakaya, vkkaya@yildiz.edu.tr

Received 29 June 2012; Revised 16 September 2012; Accepted 27 October 2012

Academic Editor: Ljubisa Kocinac

Copyright (C) 2012 Vatan Karakaya et al. This is an open access article distributed under the Creative Commons Attribution License, which permits unrestricted use, distribution, and reproduction in any medium, provided the original work is properly cited.

The purpose of this work is to investigate types of convergence of sequences of functions in intuitionistic fuzzy normed spaces and some properties related with these concepts.

\section{Introduction and Preliminaries}

The fuzzy theory has emerged as one of the most active area of research in many branches of mathematics and engineering. This new theory was introduced by Zadeh [1] in 1965 and since then a large number of research papers have appeared by using the concept of fuzzy set/numbers and fuzzification of many classical theories have also been made. It has also very useful application in various fields, for example, population dynamics [2], chaos control [3], computer programming [4], nonlinear dynamical systems [5], fuzzy physics [6], fuzzy topology $[7,8]$, and so forth. The notion of intuitionistic fuzzy sets, a generalization of fuzzy sets, was introduced by Atanassov [9] in 1986 and later there has been much progress in the study of intuitionistic fuzzy sets by many authors including Mursaleen et al. [10], Mursaleen et al. [11], and Y $ı$ lmaz [12]. Using the idea of intuitionistic fuzzy sets, Park [13] defined the notion of intuitionistic fuzzy metric spaces with the help of the continuous $t$ norms and the continuous $t$-conorms as a generalization of fuzzy metric spaces due to George and Veeramani [14]. Samanta and Jebril [15] introduced the definitions of intuitionistic fuzzy continuity and sequential intuitionistic fuzzy continuity and proved that they are equivalent. 
A few of the algebraic and topological properties of intuitionistic fuzzy continuity and uniformly intuitionistic fuzzy continuity were investigated by Dinda and Samanta [16]. On the other hand, the Fast [17] introduced the concept of statistical convergence for real number sequences. Different types of statistical convergence of sequences of real functions and related notions were first studied in [18], and some important results and references on statistical convergence and function sequences can be found in [19-23].

In this paper, primarily following the line of [18], we define statistical convergence of sequences of functions in intuitionistic fuzzy normed space (IFNS for short), and we investigate some properties related with these concepts. To explain main problems of this work, we have to give some definitions and literature acknowledgment. In [24], Schweizer and Sklar introduced a continuous $t$-norm and a continuous $t$-conorm. Afterward Saadati and Park [8] introduced the following definitions by using concepts mentioned above.

We now first recall some basic notions of intuitionistic fuzzy normed spaces.

Definition 1.1 (see [8]). Let $*$ be a continuous t-norm, $\diamond$ a continuous $t$-conorm, and $X$ a linear space over the intuitionistic fuzzy field $(\mathbb{R}$ or $\mathbb{C}$ ). If $\mu$ and $v$ are fuzzy sets on $X \times(0, \infty)$ satisfying the following conditions, the five-tuple $(X, \mu, v, *, \diamond)$ is said to be an IFNS and $(\mu, v)$ is called an intuitionistic fuzzy norm (IFN for short). For every $x, y \in X$ and $s, t>0$,

(i) $\mu(x, t)+v(x, t) \leq 1$,

(ii) $\mu(x, t)>0$,

(iii) $\mu(x, t)=1 \Leftrightarrow x=0$,

(iv) $\mu(a x, t)=\mu(x, t /|a|)$ for each $a \neq 0$,

(v) $\mu(x, t) * \mu(y, s) \leq \mu(x+y, t+s)$,

(vi) $\mu(x, \cdot):(0, \infty) \rightarrow[0,1]$ is continuous,

(vii) $\lim _{t \rightarrow \infty} \mu(x, t)=1$ and $\lim _{t \rightarrow \infty} \mu(x, t)=0$,

(viii) $v(x, t)<1$,

(ix) $v(x, t)=0 \Leftrightarrow x=0$,

(x) $v(a x, t)=v(x, t /|a|)$ for each $a \neq 0$,

(xi) $v(x, t) \diamond v(y, s) \geq v(x+y, t+s)$,

(xii) $v(x, \cdot):(0, \infty) \rightarrow[0,1]$ is continuous,

(xiii) $\lim _{t \rightarrow \infty} \mathcal{v}(x, t)=0$ and $\lim _{t \rightarrow \infty} v(x, t)=1$.

For an IFNS, we further assume that $(X, \mu, \nu, *, \diamond)$ satisfy the following axiom: (see [16])

(xiv)

$$
\left.\begin{array}{l}
a \diamond a=a \\
a * a=a
\end{array}\right\} \quad \forall a \in[0,1] .
$$

Definition 1.2 (see [8]). Let $(X, \mu, v, *, \diamond)$ be a intuitionistic fuzzy normed space. A subset $A$ of $X$ is said to be IF-bounded if there exist $t>0$ and $0<r<1$ such that $\mu(x, t)>1-r$ and $v(x, t)<r$ for each $x \in A$. 
Definition 1.3 (see [25]). Let $(X, \mu, v, *, \diamond)$ be a intuitionistic fuzzy metric space. Let $A$ be any subset of $X$. Define

$$
\phi(t)=\inf \{\mu(x, y, t): x, y \in A\}, \quad \psi(t)=\sup \{v(x, y, t): x, y \in A\},
$$

(i) $A$ is said to be $q$-bounded if $\lim _{t \rightarrow \infty} \phi(t)=1$ and $\lim _{t \rightarrow \infty} \psi(t)=0$, (ii) $A$ is said to be semibounded if $\lim _{t \rightarrow \infty} \phi(t)=k$ and $\lim _{t \rightarrow \infty} \psi(t)=1-k, 0<k<1$ (iii) $A$ is said to be unbounded if $\lim _{t \rightarrow \infty} \phi(t)=0$ and $\lim _{t \rightarrow \infty} \psi(t)=1$.

Theorem 1.4 (see [25]). Let $(X, \mu, v, *, \diamond)$ be a intuitionistic fuzzy metric space. A subset of $X$ is IF bounded if and only if $A$ is $q$-bounded or semibounded.

Definition 1.5 (see [8]). Let $(X, \mu, v, *, \diamond)$ be an IFNS and $\left(x_{k}\right)$ be a sequence in $X$. The sequence $\left(x_{k}\right)$ is said to be convergent to $L \in X$ with respect to IFN $(\mu, v)$ if for every $\varepsilon>0$ and $t>0$, there exists a positive integer $k_{0}(\varepsilon)$ such that $\mu\left(x_{k}-L, t\right)>1-\varepsilon$ and $v\left(x_{k}-L, t\right)<\varepsilon$ whenever $k>k_{0}$. In this case, we write $(\mu, v)-\lim x_{k}=L$ as $k \rightarrow \infty$.

Definition 1.6 (see [16]). Let $(X, \mu, v, *, \diamond)$ and $\left(Y, \mu^{\prime}, v^{\prime}, *, \diamond\right)$ be two IFNS. A mapping $f$ from $(X, \mu, v, *, \diamond)$ to $\left(Y, \mu^{\prime}, v^{\prime}, *, \diamond\right)$ is said to be intuitionistic fuzzy continuous at $x_{0} \in X$ if for any given $\varepsilon>0$, there exist $\delta=\delta(a, \varepsilon), \beta=\beta(a, \varepsilon) \in(0,1)$ such that for all $x \in X$ and for all $a \in(0,1)$,

$$
\begin{gathered}
\mu\left(x-x_{0}, \delta\right)>1-\beta \Longrightarrow \mu^{\prime}\left(f(x)-f\left(x_{0}\right), \varepsilon\right)>1-a, \\
v\left(x-x_{0}, \delta\right)<\beta \Longrightarrow v^{\prime}\left(f(x)-f\left(x_{0}\right), \varepsilon\right)<a .
\end{gathered}
$$

Definition 1.7 (see [16]). Let $f_{k}:(X, \mu, v, *, \diamond) \rightarrow\left(Y, \mu^{\prime}, v^{\prime}, *, \diamond\right)$ be a sequence of functions. The sequence $\left(f_{k}\right)$ is said to be pointwise intuitionistic fuzzy convergent on $X$ to a function $f$ with respect to $\left(\mu^{\prime}, v^{\prime}\right)$ if for each $x \in X$, the sequence $\left(f_{k}(x)\right)$ is convergent to $f(x)$ with respect to $\left(\mu^{\prime}, v^{\prime}\right)$.

Definition 1.8 (see [16]). Let $f_{k}:(X, \mu, v, *, \diamond) \rightarrow\left(Y, \mu^{\prime}, \nu^{\prime}, *, \diamond\right)$ be a sequence of functions. The sequence $\left(f_{k}\right)$ is said to be uniformly intuitionistic fuzzy convergent on $X$ to a function $f$ with respect to $(\mu, v)$, if given $0<r<1, t>0$, there exist a positive integer $k_{0}=k_{0}(r, t)$ such that $\forall x \in X$ and $\forall k>k_{0}$,

$$
\mu^{\prime}\left(f_{k}(x)-f(x), t\right)>1-r, \quad v^{\prime}\left(f_{k}(x)-f(x), t\right)<r .
$$

Now, we recall the notion of the statistical convergence of sequences in intuitionistic fuzzy normed spaces.

Definition 1.9 (see [26]). Let $K \subset \mathbb{N}$ and $K_{n}=\{k \in K: k \leq n\}$. Then the asymptotic density is defined by $\delta(K)=\lim _{n \rightarrow \infty}\left(\left|K_{n}\right| / n\right)$, where $\left|K_{n}\right|$ denotes the cardinality of $K_{n}$.

Definition 1.10. Let $A$ be subset of $\mathbb{N}$. If a property $P(k)$ holds for all $k \in A$ with $\delta(A)=1$, we say that $P$ holds for almost all $k(a \cdot a \cdot k)$. 
Definition 1.11 (see [26]). A sequence $x=\left(x_{k}\right)$ is said to be statistically convergent to the number $L$, or in short $s t-\lim x=L$, if for every $\varepsilon>0$, the set $K(\varepsilon)$ has asymptotic density zero, where

$$
K(\varepsilon)=\left\{k \in \mathbb{N}:\left|x_{k}-L\right| \geq \varepsilon\right\},
$$

that is,

$$
\left|x_{k}-L\right|<\varepsilon \quad a \cdot a \cdot k \text {. }
$$

Definition 1.12 (see [23]). Let $(X, \mu, v, *, \diamond)$ be an IFNS. Then, sequence $\left(x_{k}\right)$ is said to be statistically convergent to $L \in X$ with respect to $\operatorname{IFN}(\mu, v)$ provided that for every $\varepsilon>0$ and $t>0$,

$$
\delta\left(\left\{k \in \mathbb{N}: \mu\left(x_{k}-L, t\right) \leq 1-\varepsilon \text { or } v\left(x_{k}-L, t\right) \geq \varepsilon\right\}\right)=0,
$$

or equivalently

$$
\lim _{n \rightarrow \infty} \frac{1}{n} \mid\left\{k \leq n: \mu\left(x_{k}-L, t\right) \leq 1-\varepsilon \text { or } v\left(x_{k}-L, t\right) \geq \varepsilon\right\} \mid=0 .
$$

In this case, we write $s t_{\mu, v}-\lim \left(x_{k}\right)=L$.

Definition 1.13 (see [23]). Let $(X, \mu, v, *, \diamond)$ be an IFNS. Sequence $\left(x_{k}\right)$ is said to be statistically Cauchy with respect to IFN $(\mu, v)$ provided that for every $\varepsilon>0$ and $t>0$, there exists a number $m \in \mathbb{N}$ satisfying

$$
\delta\left(\left\{k \in \mathbb{N}: \mu\left(x_{k}-x_{m}, t\right) \leq 1-\varepsilon \text { or } v\left(x_{k}-x_{m}, t\right) \geq \varepsilon\right\}\right)=0 .
$$

\section{Statistical Convergence of Sequences of Functions in Intuitionistic Fuzzy Normed Spaces}

In this section, we define pointwise statistically and uniformly statistically convergent sequences of functions in intuitionistic fuzzy normed spaces. Also, we give the statistical analog of the Cauchy convergence criterion for pointwise and uniformly statistical convergent in intuitionistic fuzzy normed space. Finally, we prove that uniformly statistical convergence preserves continuity.

Definition 2.1. Let $(X, \mu, v, *, \diamond)$ and $\left(Y, \mu^{\prime}, v^{\prime}, *, \diamond\right)$ be two IFNS and $f_{k}:(X, \mu, v, *, \diamond) \rightarrow$ $\left(Y, \mu^{\prime}, v^{\prime}, *, \diamond\right)$ a sequence of functions. We say that a sequence $\left(f_{k}\right)$ pointwise statistically converges to $f$ with respect to intuitionistic fuzzy norm $\left(\mu^{\prime}, v^{\prime}\right)$ if $f_{k}(x)$ statistically converges to $f(x)$ for each $x \in X$ with respect to intuitionistic fuzzy norm $\left(\mu^{\prime}, v^{\prime}\right)$ and we write $s t_{\mu, \nu}-f_{k} \rightarrow f$.

Theorem 2.2. Let $f_{k}:(X, \mu, v, *, \diamond) \rightarrow\left(Y, \mu^{\prime}, v^{\prime}, *, \diamond\right)$ be a sequence of functions. If $\left(f_{k}\right)$ is pointwise intuitionistic fuzzy convergent on $X$ with respect to $(\mu, v)$, then $s t_{\mu, v}-f_{k} \rightarrow f$. But the converse of this is not true. 
Proof. Let $\left(f_{k}\right)$ be pointwise intuitionistic fuzzy convergent on $X$. In this case the sequence $\left(f_{k}(x)\right)$ is convergent with respect to $\left(\mu^{\prime}, v^{\prime}\right)$ for each $x \in X$. Then for each $\varepsilon>0$ and $t>0$, there is number $k_{0}(\varepsilon) \in \mathbb{N}$ such that

$$
\mu^{\prime}\left(f_{k}(x)-f(x), t\right)>1-\varepsilon, \quad v^{\prime}\left(f_{k}(x)-f(x), t\right)<\varepsilon
$$

for all $k \geq k_{0}$ and for each $x \in X$. Hence for each $x \in X$ the set

$$
\left\{k \in \mathbb{N}: \mu^{\prime}\left(f_{k}(x)-f(x), t\right) \leq 1-\varepsilon \text { or } v^{\prime}\left(f_{k}(x)-f(x), t\right) \geq \varepsilon\right\}
$$

has finite numbers of terms. Since density of finite subset of $\mathbb{N}$ is 0 , hence

$$
\delta\left(\left\{k \in \mathbb{N}: \mu^{\prime}\left(f_{k}(x)-f(x), t\right) \leq 1-\varepsilon \text { or } v^{\prime}\left(f_{k}(x)-f(x), t\right) \geq \varepsilon\right\}\right)=0 .
$$

That is, $s t_{\mu, v}-f_{k} \rightarrow f$.

Example 2.3. Let $(\mathbb{R},|\cdot|)$ denote the space of real numbers with the usual norm, and let $a * b=$ $a \cdot b$ and $a \diamond b=\min \{a+b, 1\}$ for $a, b \in[0,1]$. For all $x \in \mathbb{R}$ and every $t>0$, we consider

$$
\mu(x, t)=\frac{t}{t+|x|}, \quad v(x, t)=\frac{|x|}{t+|x|} .
$$

In this case, $(\mathbb{R}, \mu, v, *, \diamond)$ is an IFNS (also, $([0,1], \mu, v, *, \diamond)$ is intuitionistic fuzzy normed space.). Let $f_{k}:[0,1] \rightarrow \mathbb{R}$ be a sequence of functions whose terms are given by

$$
f_{k}(x)=\left\{\begin{array}{lll}
x^{k^{2}}+1 & \text { if } k=m^{2} & (m \in \mathbb{N}), x \in\left[0, \frac{1}{2}\right), \\
0 & \text { if } k \neq m^{2} \quad(m \in \mathbb{N}), x \in\left[0, \frac{1}{2}\right), \\
0 & \text { if } k=m^{2} \quad(m \in \mathbb{N}), \quad x \in\left[\frac{1}{2}, 1\right), \\
x^{k}+\frac{1}{2} & \text { if } k \neq m^{2} \quad(m \in \mathbb{N}), \quad x \in\left[\frac{1}{2}, 1\right), \\
2 & \text { if } x=1 .
\end{array}\right.
$$


Then sequence $\left(f_{k}\right)$ is pointwise statistically intuitionistic convergent on $[0,1]$ with respect to $(\mu, v)$. Indeed, for $x \in[0,1 / 2)$, since

$$
\begin{aligned}
K_{n}(\varepsilon, t)= & \left\{k \leq n: \mu\left(f_{k}(x)-f(x), t\right) \leq 1-\varepsilon \text { or } v\left(f_{k}(x)-f(x), t\right) \geq \varepsilon\right\}, \\
K_{n}(\varepsilon, t) & =\left\{k \leq n: \frac{t}{t+\left|f_{k}(x)-0\right|} \leq 1-\varepsilon \text { or } \frac{\left|f_{k}(x)-0\right|}{t+\left|f_{k}(x)-0\right|} \geq \varepsilon\right\} \\
& =\left\{k \leq n:\left|f_{k}(x)\right| \geq \frac{\varepsilon t}{1-\varepsilon}\right\} \\
& =\left\{k \leq n: f_{k}(x)=x^{k^{2}}+1\right\} \\
& =\left\{k \leq n: k=m^{2}, m \in \mathbb{N}\right\}
\end{aligned}
$$

we have

$$
\delta\left(K_{n}(\varepsilon, t)\right)=\frac{1}{n}\left|\left\{k \leq n: k=m^{2}, m \in \mathbb{N}\right\}\right| \leq \frac{\sqrt{n}}{n},
$$

which yields $\lim _{n \rightarrow \infty} \delta\left(K_{n}(\varepsilon, t)\right)=0$. Thus, for each $x \in[0,1 / 2)$, sequence $\left(f_{k}\right)$ is statistically convergent to 0 with respect to intuitionistic fuzzy norm $(\mu, v)$.

If we take $x \in[1 / 2,1)$, then we have

$$
\begin{aligned}
K_{n}^{\prime}(\varepsilon, t) & =\left\{k \leq n: \frac{t}{t+\left|f_{k}(x)-(1 / 2)\right|} \leq 1-\varepsilon \text { or } \frac{\left|f_{k}(x)-(1 / 2)\right|}{t+\left|f_{k}(x)-(1 / 2)\right|} \geq \varepsilon\right\} \\
& =\left\{k \leq n:\left|f_{k}(x)-\frac{1}{2}\right| \geq \frac{\varepsilon t}{1-\varepsilon}\right\} \\
& =\left\{k \leq n: k=m^{2},\left|f_{k}(x)-\frac{1}{2}\right| \geq \frac{\varepsilon t}{1-\varepsilon}\right\} \cup\left\{k \leq n: k \neq m^{2},\left|f_{k}(x)-\frac{1}{2}\right| \geq \frac{\varepsilon t}{1-\varepsilon}\right\} \\
& =\left\{k \leq n: k=m^{2}\right\} \cup\left\{k \leq n: k \neq m^{2}:\left|x^{k}+\frac{1}{2}-\frac{1}{2}\right| \geq \frac{\varepsilon t}{1-\varepsilon}\right\} \\
& =\left\{k \leq n: k=m^{2}\right\} \cup\left\{k \leq n: k \neq m^{2}:\left|x^{k}\right| \geq \frac{\varepsilon t}{1-\varepsilon}\right\} .
\end{aligned}
$$

Therefore, density of $K_{n}^{\prime}(\varepsilon, t)$ is 0 and for each $x \in[1 / 2,1)$, sequence $\left(f_{k}\right)$ is statistically convergent to $1 / 2$ with respect to IFN $(\mu, v)$. If we take $x=1$, it can be seen easily that $\left(f_{k}\right)$ is 
intuitionistic fuzzy convergent to 2 . Hence $\left(f_{k}\right)$ is intuitionistic fuzzy statistically convergent to 2 . That is

$$
s t_{\mu, v}-f_{k}(x)= \begin{cases}0, & x \in\left[0, \frac{1}{2}\right) \\ \frac{1}{2}, & x \in\left[\frac{1}{2}, 1\right) \\ 2, & x=1 .\end{cases}
$$

Since $f_{k}(x)$ is statistically convergent to different points with respect to intuitionistic fuzzy norm $(\mu, v)$ for each $x \in X$, it can be seen that $\left(f_{k}\right)$ is pointwise statistically intuitionistic fuzzy convergent on $[0,1]$.

Lemma 2.4. Let $f_{k}:(X, \mu, v, *, \diamond) \rightarrow\left(Y, \mu^{\prime}, v^{\prime}, *, \diamond\right)$ be sequence of functions. Then the following statements are equivalent:

(i) $s t_{\mu, v}-f_{k} \rightarrow f$,

(ii) $\delta\left\{k \in \mathbb{N}: \mu^{\prime}\left(f_{k}(x)-f(x), t\right) \leq 1-\varepsilon\right\}=\delta\left\{k \in \mathbb{N}: v^{\prime}\left(f_{k}(x)-f(x), t\right) \geq \varepsilon\right\}=0$ for each $x \in X$, for each $\varepsilon>0$ and $t>0$,

(iii) $\delta\left\{k \in \mathbb{N}: \mu^{\prime}\left(f_{k}(x)-f(x), t\right)>1-\varepsilon\right.$ and $\left.\nu^{\prime}\left(f_{k}(x)-f(x), t\right)<\varepsilon\right\}=1$ for each $x \in X$, for each $\varepsilon>0$ and $t>0$,

(iv) $s t-\lim \mu^{\prime}\left(f_{k}(x)-f(x), t\right)=1$ and $s t-\lim v^{\prime}\left(f_{k}(x)-f(x), t\right)=0$ for each $x \in X$ and $t>0$.

Theorem 2.5. Let $\left(f_{k}\right)$ and $\left(g_{k}\right)$ be two sequences of functions from $(X, \mu, v, *, \diamond)$ to $\left(Y, \mu^{\prime}, v^{\prime}, *, \diamond\right)$. If $s t_{\mu, v}-f_{k} \rightarrow f$ and $s t_{\mu, v}-g_{k} \rightarrow g$, then $s t_{\mu, v}-\left(\alpha f_{k}+\beta g_{k}\right) \rightarrow \alpha f+\beta g$ where $\alpha, \beta \in(\mathbb{R}$ or $\mathbb{C})$.

Proof. The proof is clear for $\alpha=0$ and $\beta=0$. Now let $\alpha \neq 0$ and $\beta \neq 0$. Since $s t_{\mu, v}-f_{k} \rightarrow f$ and $s t_{\mu, v}-g_{k} \rightarrow g$, for each $x \in X$, if we define

$$
\begin{gathered}
A_{1}=\left\{k \in \mathbb{N}: \mu^{\prime}\left(f_{k}(x)-f(x), \frac{t}{2|\alpha|}\right) \leq 1-\varepsilon \text { or } v^{\prime}\left(f_{k}(x)-f(x), \frac{t}{2|\alpha|}\right) \geq \varepsilon\right\}, \\
A_{2}=\left\{k \in \mathbb{N}: \mu^{\prime}\left(g_{k}(x)-g(x), \frac{t}{2|\beta|}\right) \leq 1-\varepsilon \text { or } v^{\prime}\left(g_{k}(x)-g(x), \frac{t}{2|\beta|}\right) \geq \varepsilon\right\},
\end{gathered}
$$

then

$$
\delta\left(A_{1}\right)=0, \quad \delta\left(A_{2}\right)=0 .
$$

Since $\delta\left(A_{1}\right)=0$ and $\delta\left(A_{2}\right)=0$, if we state $A$ by $\left(A_{1} \cup A_{2}\right)$ then

$$
\delta(A)=0
$$


Hence, $A_{1} \cup A_{2} \neq \mathbb{N}$ and there exists $\exists m \in \mathbb{N}$ such that

$$
\begin{array}{cc}
\mu^{\prime}\left(f_{m}(x)-f(x), \frac{t}{2|\alpha|}\right)>1-\varepsilon, & v^{\prime}\left(f_{m}(x)-f(x), \frac{t}{2|\alpha|}\right)<\varepsilon, \\
\mu^{\prime}\left(g_{m}(x)-g(x), \frac{t}{2|\beta|}\right)>1-\varepsilon, & v^{\prime}\left(g_{m}(x)-g(x), \frac{t}{2|\beta|}\right)<\varepsilon .
\end{array}
$$

Let

$$
\begin{gathered}
B=\left\{k \in \mathbb{N}: \mu^{\prime}\left(\left(\alpha f_{k}+\beta g_{k}\right)(x)-(\alpha f(x)+\beta g(x)), t\right)>1-\varepsilon,\right. \\
\left.v^{\prime}\left(\left(\alpha f_{k}+\beta g_{k}\right)(x)-(\alpha f(x)+\beta g(x)), t\right)<\varepsilon\right\} .
\end{gathered}
$$

We will show that for each $x \in X$

$$
A^{c} \subset B .
$$

Let $m \in A^{c}$. In this case,

$$
\begin{array}{cc}
\mu^{\prime}\left(f_{m}(x)-f(x), \frac{t}{2|\alpha|}\right)>1-\varepsilon, & v^{\prime}\left(f_{m}(x)-f(x), \frac{t}{2|\alpha|}\right)<\varepsilon, \\
\mu^{\prime}\left(g_{m}(x)-g(x), \frac{t}{2|\beta|}\right)>1-\varepsilon, & v^{\prime}\left(g_{m}(x)-g(x), \frac{t}{2|\beta|}\right)<\varepsilon .
\end{array}
$$

Using those above, we have

$$
\begin{aligned}
\mu^{\prime}\left(\left(\alpha f_{m}+\beta g_{m}\right)(x)-(\alpha f+\beta g)(x), t\right) & \geq \mu^{\prime}\left(\alpha f_{m}(x)-\alpha f(x), \frac{t}{2}\right) * \mu^{\prime}\left(\beta g_{m}(x)-\beta g(x), \frac{t}{2}\right) \\
& =\mu^{\prime}\left(f_{m}(x)-f(x), \frac{t}{2|\alpha|}\right) * \mu^{\prime}\left(g_{m}(x)-g(x), \frac{t}{2|\beta|}\right) \\
& >(1-\varepsilon) *(1-\varepsilon) \\
& =(1-\varepsilon), \\
v^{\prime}\left(\left(\alpha f_{m}+\beta g_{m}\right)(x)-(\alpha f+\beta g)(x), t\right) & \leq v^{\prime}\left(\alpha f_{m}(x)-\alpha f(x), \frac{t}{2}\right) * v^{\prime}\left(\beta g_{m}(x)-\beta g(x), \frac{t}{2}\right) \\
& =v^{\prime}\left(f_{m}(x)-f(x), \frac{t}{2|\alpha|}\right) * v^{\prime}\left(g_{m}(x)-g(x), \frac{t}{2|\beta|}\right) \\
& <\varepsilon \diamond \varepsilon \\
& =\varepsilon .
\end{aligned}
$$


This implies that

$$
A^{c} \subset B .
$$

Since $B^{c} \subset A$ and $\delta(A)=0$, hence

$$
\delta\left(B^{c}\right)=0,
$$

that is

$$
\begin{gathered}
\delta\left(\left\{k \in \mathbb{N}: \mu^{\prime}\left(\left(\alpha f_{k}+\beta g_{k}\right)(x)-(\alpha f+\beta g)(x), t\right) \leq 1-\varepsilon,\right.\right. \\
\left.\left.v^{\prime}\left(\left(\alpha f_{k}+\beta g_{k}\right)(x)-(\alpha f+\beta g)(x), t\right) \geq \varepsilon\right\}\right)=0
\end{gathered}
$$

which means

$$
s t_{\mu, v}-\left(\alpha f_{k}+\beta g_{k}\right) \longrightarrow \alpha f+\beta g
$$

Definition 2.6. Let $f_{k}:(X, \mu, v, *, \diamond) \rightarrow\left(Y, \mu^{\prime}, v^{\prime}, *, \diamond\right)$ be a sequence of functions. The sequence $\left(f_{k}\right)$ is a pointwise statistically Cauchy sequence in IFNS provided that for each $\varepsilon>0$ and $t>0$ there exists $N=N(\varepsilon, t, x)$ such that

$$
\delta\left(\left\{k \in \mathbb{N}: \mu^{\prime}\left(f_{k}(x)-f_{N}(x), t\right) \leq 1-\varepsilon \text { or } v^{\prime}\left(f_{k}(x)-f_{N}(x), t\right) \geq \varepsilon \text { for each } x \in X\right\}\right)=0 .
$$

That is, there exists a number $N=N(\varepsilon, t, x)$ for each $x \in X$ such that

$$
\mu^{\prime}\left(f_{k}(x)-f_{N}(x), t\right)>1-\varepsilon, v^{\prime}\left(f_{k}(x)-f_{N}(x), t\right)<\varepsilon \quad \text { for } a \cdot a \cdot k .
$$

Theorem 2.7. Let $f_{k}:(X, \mu, v, *, \diamond) \rightarrow\left(Y, \mu^{\prime}, v^{\prime}, *, \diamond\right)$ be a sequence of functions. If $\left(f_{k}\right)$ is a pointwise statistically convergent sequence with respect to intuitionistic fuzzy norm $(\mu, v)$, then $\left(f_{k}\right)$ is a pointwise statistically Cauchy sequence with respect to intuitionistic fuzzy norm $(\mu, v)$.

Proof. Suppose that $s t_{\mu, v}-f_{k} \rightarrow f$ and let $\varepsilon>0, t>0$. For given each $\varepsilon>0$, choose $s>0$ such that $(1-\varepsilon) *(1-\varepsilon)>1-s$ and $\varepsilon \diamond \varepsilon<s$. If we state, respectively, $A_{x}(\varepsilon, t)$ and $A_{x}^{c}(\varepsilon, t)$ by

$$
\begin{gathered}
\left\{k \in \mathbb{N}: \mu^{\prime}\left(f_{k}(x)-f(x), \frac{t}{2}\right) \leq 1-\varepsilon \text { or } v^{\prime}\left(f_{k}(x)-f(x), \frac{t}{2}\right) \geq \varepsilon\right\}, \\
\left\{k \in \mathbb{N}: \mu^{\prime}\left(f_{k}(x)-f(x), \frac{t}{2}\right)>1-\varepsilon, v^{\prime}\left(f_{k}(x)-f(x), \frac{t}{2}\right)<\varepsilon\right\},
\end{gathered}
$$

for each $x \in X$. Then, we have

$$
\delta\left(A_{x}(\varepsilon, t)\right)=0,
$$


which implies that

$$
\delta\left(A_{x}^{c}(\varepsilon, t)\right)=1
$$

Let $N \in A_{x}^{c}(\varepsilon, t)$. Then

$$
\mu^{\prime}\left(f_{N}(x)-f(x), \frac{t}{2}\right)>1-\varepsilon, \quad v^{\prime}\left(f_{N}(x)-f(x), \frac{t}{2}\right)<\varepsilon
$$

We want to show that there exists a number $N=N(x, \varepsilon, t)$ such that

$$
\delta\left(\left\{k \in \mathbb{N}: \mu^{\prime}\left(f_{k}(x)-f_{N}(x), t\right) \leq 1-s \text { or } v^{\prime}\left(f_{k}(x)-f_{N}(x), t\right) \geq s \text { for each } x \in X\right\}\right)=0
$$

Therefore, define for each $x \in X$,

$$
B_{x}(\varepsilon, t)=\left\{k \in \mathbb{N}: \mu^{\prime}\left(f_{k}(x)-f_{N}(x), t\right) \leq 1-s \text { or } v^{\prime}\left(f_{k}(x)-f_{N}(x), t\right) \geq s\right\} .
$$

We have to show that

$$
B_{x}(\varepsilon, t) \subset A_{x}(\varepsilon, t) .
$$

Suppose that

$$
B_{x}(\varepsilon, t) \nsubseteq A_{x}(\varepsilon, t)
$$

In this case, $B_{x}(\varepsilon, t)$ has at least one different element which $A_{x}(\varepsilon, t)$ does not has. Let $k \in$ $B_{x}(\varepsilon, t) \backslash A_{x}(\varepsilon, t)$. Then we have

$$
\mu^{\prime}\left(f_{k}(x)-f_{N}(x), t\right) \leq 1-s, \quad \mu^{\prime}\left(f_{k}(x)-f(x), \frac{t}{2}\right)>1-\varepsilon,
$$

in particularly $\mu^{\prime}\left(f_{N}(x)-f(x), t / 2\right)>1-\varepsilon$. In this case,

$$
\begin{aligned}
1-s & \geq \mu^{\prime}\left(f_{k}(x)-f_{N}(x), t\right) \geq \mu^{\prime}\left(f_{k}(x)-f(x), \frac{t}{2}\right) * \mu^{\prime}\left(f_{N}(x)-f(x), \frac{t}{2}\right) \\
& \geq(1-\varepsilon) *(1-\varepsilon)>1-s,
\end{aligned}
$$

which is not possible. On the other hand

$$
v^{\prime}\left(f_{k}(x)-f_{N}(x), t\right) \geq s, \quad v^{\prime}\left(f_{k}(x)-f(x), t\right)<\varepsilon,
$$


in particularly $v^{\prime}\left(f_{N}(x)-f(x), t\right)<\varepsilon$. In this case,

$$
\begin{aligned}
s & \leq v^{\prime}\left(f_{k}(x)-f_{N}(x), t\right) \leq v^{\prime}\left(f_{k}(x)-f(x), \frac{t}{2}\right) \diamond v^{\prime}\left(f_{N}(x)-f(x), \frac{t}{2}\right) \\
& <\varepsilon \diamond \varepsilon<s
\end{aligned}
$$

which is not possible. Hence $B_{x}(\varepsilon, t) \subset A_{x}(\varepsilon, t)$. Therefore, by $\delta\left(A_{x}(\varepsilon, t)\right)=0, \delta\left(B_{x}(\varepsilon, t)\right)=0$. That is, $\left(f_{k}\right)$ is a pointwise statistical Cauchy sequence with respect to intuitionistic fuzzy norm $(\mu, v)$.

Afterward this step, we introduce a uniformly statistical convergence of sequences of function in an IFNS. To do this, we need the following definition

Definition 2.8. Let $(X, \mu, v, *, \diamond)$ and $\left(Y, \mu^{\prime}, v^{\prime}, *, \diamond\right)$ be two intuitionistic fuzzy normed linear space over the same field IF and $f_{k}:(X, \mu, \nu, *, \diamond) \rightarrow\left(Y, \mu^{\prime}, \nu^{\prime}, *, \diamond\right)$ be a sequence of functions. $\left(f_{k}\right)$ converges uniform statistically to $f$ with respect to $(\mu, v) \Leftrightarrow \forall \varepsilon>0, \exists M \subset \mathbb{N}, \delta(M)=1$ and $\exists k_{0}=k_{0}(\varepsilon, t) \in M \ni \forall k>k_{0}$ and $k \in M$ and $\forall x \in X$,

$$
\mu^{\prime}\left(f_{k}(x)-f(x), t\right)>1-\varepsilon, \quad v^{\prime}\left(f_{k}(x)-f(x), t\right)<\varepsilon .
$$

Lemma 2.9. Let $f_{k}:(X, \mu, v, *, \diamond) \rightarrow\left(Y, \mu^{\prime}, v^{\prime}, *, \diamond\right)$ be a sequence of functions. Then the following statements are equivalent:

(i) $s t_{\mu, v}-f_{k} \rightrightarrows f$.

(ii) $\delta\left\{k \in \mathbb{N}: \mu^{\prime}\left(f_{k}(x)-f(x), t\right) \leq 1-\varepsilon\right\}=\delta\left\{k \in \mathbb{N}: v^{\prime}\left(f_{k}(x)-f(x), t\right) \geq \varepsilon\right\}=0$ for all $x \in X$, for every $\varepsilon>0$ and $t>0$.

(iii) $\delta\left\{k \in \mathbb{N}: \mu^{\prime}\left(f_{k}(x)-f(x), t\right)>1-\varepsilon\right.$ and $\left.v^{\prime}\left(f_{k}(x)-f(x), t\right)<\varepsilon\right\}=1$ for all $x \in X$, for every $\varepsilon>0$ and $t>0$.

(iv) $s t-\lim \mu^{\prime}\left(f_{k}(x)-f(x), t\right)=1$ and st $-\lim v^{\prime}\left(f_{k}(x)-f(x), t\right)=0$ for all $x \in X$ and $t>0$.

Proposition 2.10. Let the sequence $\left(f_{k}\right)$ and $f$ be bounded functions from $(X, \mu, \nu, *, \diamond)$ to $\left(Y, \mu^{\prime}, v^{\prime}, *, \diamond\right) . f_{k}$ is intuitionistic fuzzy uniformly statistically convergent to $f$ if and only if

$$
s t-\lim \inf \mu^{\prime}\left(f_{k}(x)-f(x), t\right)=1, \quad s t-\lim \sup v^{\prime}\left(f_{k}(x)-f(x), t\right)=0,
$$

where the supremum and infimum are taken over all $x \in X$.

Proof. Suppose that $s t_{\mu, v}-f_{k} \rightrightarrows f$ on $X$. Since $\left(f_{k}\right)$ and $f$ are bounded in $(X, \mu, \nu, *, \diamond)$ for each $k \in \mathbb{N}$, by using Definition 1.3 and Theorem 1.4, we have inf $\mu^{\prime}\left(f_{k}(x)-f(x), t\right)=r_{k}$ and sup $v^{\prime}\left(f_{k}(x)-f(x), t\right)=1-r_{k}$ for each $k \in \mathbb{N}$ and for each $t>0,\left(0<r_{k}<1\right)$. By using (iv) of Lemma 2.9, we get

$$
s t-\lim \inf \mu^{\prime}\left(f_{k}(x)-f(x), t\right)=1, \quad s t-\lim \sup v^{\prime}\left(f_{k}(x)-f(x), t\right)=0,
$$

where the supremum and infimum are taken over all $x \in X$. 
Conversely, suppose that

$$
s t-\lim \inf \mu^{\prime}\left(f_{k}(x)-f(x), t\right)=1, \quad s t-\lim \sup v^{\prime}\left(f_{k}(x)-f(x), t\right)=0,
$$

where the supremum and infimum are taken over all $x \in X$. Since

$$
\inf \mu^{\prime}\left(f_{k}(x)-f(x), t\right) \in(0,1], \quad \sup v^{\prime}\left(f_{k}(x)-f(x), t\right) \in[0,1),
$$

from definition statistical convergence, for $a \cdot a \cdot k$, for every $\varepsilon>0$ and $t>0$

$$
\left|\inf \mu^{\prime}\left(f_{k}(x)-f(x), t\right)-1\right|<\varepsilon, \quad\left|\sup v^{\prime}\left(f_{k}(x)-f(x), t\right)-0\right|<\varepsilon \text {. }
$$

For all $x \in X$

$$
\begin{aligned}
\mu^{\prime}\left(f_{k}(x)-f(x), t\right) & \geq \inf \mu^{\prime}\left(f_{k}(x)-f(x), t\right) \\
& \Longrightarrow-\mu^{\prime}\left(f_{k}(x)-f(x), t\right)<-\inf \mu^{\prime}\left(f_{k}(x)-f(x), t\right) \\
& \Longrightarrow 1-\mu^{\prime}\left(f_{k}(x)-f(x), t\right)<1-\inf \mu^{\prime}\left(f_{k}(x)-f(x), t\right)
\end{aligned}
$$

since $\mu^{\prime}\left(f_{k}(x)-f(x), t\right) \in(0,1] \forall x \in X$

$$
\begin{aligned}
& \Longrightarrow\left|1-\mu^{\prime}\left(f_{k}(x)-f(x), t\right)\right|<\left|1-\inf \mu^{\prime}\left(f_{k}(x)-f(x), t\right)\right| \\
& \Longrightarrow\left|1-\mu^{\prime}\left(f_{k}(x)-f(x), t\right)\right|<\left|1-\inf \mu^{\prime}\left(f_{k}(x)-f(x), t\right)\right|<\varepsilon \\
& \Longrightarrow\left|1-\mu^{\prime}\left(f_{k}(x)-f(x), t\right)\right|<\varepsilon \quad a \cdot a \cdot k, \\
& v^{\prime}\left(f_{k}(x)-f(x), t\right) \leq \sup v^{\prime}\left(f_{k}(x)-f(x), t\right) \\
& \Longrightarrow\left|v^{\prime}\left(f_{k}(x)-f(x), t\right)-0\right| \leq\left|\sup v^{\prime}\left(f_{k}(x)-f(x), t\right)-0\right|<\varepsilon \\
& \Longrightarrow\left|v^{\prime}\left(f_{k}(x)-f(x), t\right)-0\right|<\varepsilon \quad a \cdot a \cdot k .
\end{aligned}
$$

Therefore, $s t-\lim \mu^{\prime}\left(f_{k}(x)-f(x), t\right)=1$ and $s t-\lim v^{\prime}\left(f_{k}(x)-f(x), t\right)=0$ for all $x \in X$ and $t>0$. From Lemma 2.9, we get $s t_{\mu, \nu}-f_{k} \rightrightarrows f$.

Example 2.11. Let $(\mathbb{R}, \mu, v, *, \diamond)$ be as Example 2.3. Consider $f_{k}:[0,1) \rightarrow \mathbb{R}$ be sequence of functions whose terms are given by

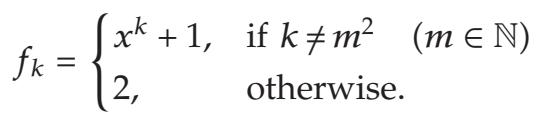

Then, for every $0<\varepsilon<1$ and for every $t>0$, we define

$$
K_{n}=\left\{k \leq n: \mu\left(f_{k}(x)-f(x), t\right) \leq 1-\varepsilon \text { or } v\left(f_{k}(x)-f(x), t\right) \geq \varepsilon\right\} .
$$


For all $x \in X$, we have

$$
\delta\left(K_{n}\right)=\delta\left(\left\{k \leq n: \mu\left(f_{k}(x)-1, t\right) \leq 1-\varepsilon \text { or } v\left(f_{k}(x)-1, t\right) \geq \varepsilon\right\}\right)=0 .
$$

Since

$$
s t_{\mu, v}-f_{k}(x)=1
$$

for all $x \in X$, the sequence $\left(f_{k}\right)$ is uniformly statistically intuitionistic fuzzy convergent to 1 on $[0,1)$.

Example 2.12. Let $(\mathbb{R}, \mu, v, *, \diamond)$ be as Example 2.3. Consider $f_{k}:[-1,1] \rightarrow \mathbb{R}$ be sequence of functions whose terms are given by

$$
f_{k}= \begin{cases}\left(x-\frac{1}{k}\right)^{2}, & \text { if } k \neq m^{2} \quad(m \in \mathbb{N}) \\ 2, & \text { otherwise }\end{cases}
$$

Since $s t_{\mu, \nu}-f_{k}(x)=x^{2}$ for all $x \in[-1,1], f_{k}$ is uniformly statistically intuitionistic fuzzy convergent to $x^{2}$ on $[-1,1]$. We can show this using Proposition 2.10 as the following, since $f_{k}$ is bounded functions sequence on $[-1,1]$.

We want to find

$$
\begin{aligned}
& \lim _{k \rightarrow \infty} \inf _{x \in[-1,1]} \mu\left(f_{k}(x)-f(x), t\right)=\frac{t}{t+\left|-(2 x / k)+\left(1 / k^{2}\right)\right|}, \\
& \lim _{k \rightarrow \infty} \sup _{x \in[-1,1]} v\left(f_{k}(x)-f(x), t\right)=\frac{\left|-(2 x / k)+1 / k^{2}\right|}{t+\left|-(2 x / k)+\left(1 / k^{2}\right)\right|} .
\end{aligned}
$$

Firstly, we need to find $\lim _{k \rightarrow \infty} \inf \mu\left(f_{k}(x)-f(x), t\right)$ and $\lim _{k \rightarrow \infty} \sup v\left(f_{k}(x)-f(x), t\right)$ for over all $x \in[-1,1 / 2 k]$ and for over all $x \in(1 / 2 k, 1]$, respectively. In case of $x \in[-1,1 / 2 k]$, we have $-(2 x / k)+\left(1 / k^{2}\right) \geq 0$. Since

$$
\begin{aligned}
0 \leq-\frac{2 x}{k}+\frac{1}{k^{2}} \leq \frac{2}{k}+\frac{1}{k^{2}} & \Longrightarrow t \leq t-\frac{2 x}{k}+\frac{1}{k^{2}} \leq t+\frac{2}{k}+\frac{1}{k^{2}} \\
& \Longrightarrow \frac{1}{t+(2 / k)+\left(1 / k^{2}\right)} \leq \frac{1}{t-(2 x / k)+\left(1 / k^{2}\right)} \leq \frac{1}{t} \\
& \Longrightarrow \frac{t}{t+(2 / k)+\left(1 / k^{2}\right)} \leq \frac{t}{t-(2 x / k)+\left(1 / k^{2}\right)} \leq 1
\end{aligned}
$$

we get

$$
\begin{aligned}
\lim _{k \rightarrow \infty} \inf _{x \in[-1,1 / 2 k]} \mu\left(f_{k}(x)-x^{2}, t\right) & =\lim _{k \rightarrow \infty} \inf _{x \in[-1,1 / 2 k]} \frac{t}{t-(2 x / k)+\left(1 / k^{2}\right)} \\
& =\lim _{k \rightarrow \infty} \frac{t}{t+(2 / k)+\left(1 / k^{2}\right)}=1 .
\end{aligned}
$$


On the other hand, we have

$$
\frac{-(2 x / k)+\left(1 / k^{2}\right)}{t-(2 x / k)+\left(1 / k^{2}\right)} \leq \frac{(2 / k)+\left(1 / k^{2}\right)}{t}
$$

and so

$$
\begin{aligned}
\lim _{k \rightarrow \infty} \sup _{x \in[-1,1 / 2 k]} v\left(f_{k}(x)-x^{2}, t\right) & =\lim _{k \rightarrow \infty} \sup _{x \in[-1,1 / 2 k]} \frac{-(2 x / k)+\left(1 / k^{2}\right)}{t-(2 x / k)+\left(1 / k^{2}\right)} \\
& =\lim _{k \rightarrow \infty} \frac{(2 / k)+\left(1 / k^{2}\right)}{t}=0 .
\end{aligned}
$$

In case of $x \in(1 / 2 k, 1]$, we have $-(2 x / k)+\left(1 / k^{2}\right)<0$. Since

$$
\begin{aligned}
-\frac{2}{k}+\frac{1}{k^{2}}<-\frac{2 x}{k}+\frac{1}{k^{2}} \leq 0 & \Longrightarrow 0 \leq \frac{2 x}{k}-\frac{1}{k^{2}}<\frac{2}{k}-\frac{1}{k^{2}} \\
& \Longrightarrow t<t+\frac{2 x}{k}-\frac{1}{k^{2}} \leq t+\frac{2}{k}-\frac{1}{k^{2}} \\
& \Longrightarrow \frac{1}{t+(2 / k)-\left(1 / k^{2}\right)}<\frac{1}{t+(2 x / k)-\left(1 / k^{2}\right)} \leq \frac{1}{t} \\
& \Longrightarrow \frac{t}{t+(2 / k)-\left(1 / k^{2}\right)} \leq \frac{t}{t+(2 x / k)-\left(1 / k^{2}\right)}<1
\end{aligned}
$$

we get

$$
\begin{aligned}
\lim _{k \rightarrow \infty} \inf _{x \in(1 / 2 k, 1]} \mu\left(f_{k}(x)-x^{2}, t\right) & =\lim _{k \rightarrow \infty} \inf _{x \in(1 / 2 k, 1]} \frac{t}{t+(2 x / k)-\left(1 / k^{2}\right)} \\
& =\lim _{k \rightarrow \infty} \frac{t}{t+(2 / k)-\left(1 / k^{2}\right)}=1 .
\end{aligned}
$$

On the other hand, we have

$$
\frac{(2 x / k)-\left(1 / k^{2}\right)}{t+(2 x / k)-\left(1 / k^{2}\right)} \leq \frac{(2 / k)-\left(1 / k^{2}\right)}{t}
$$

and so

$$
\begin{aligned}
\lim _{k \rightarrow \infty} \sup _{x \in(1 / 2 k, 1]} v\left(f_{k}(x)-x^{2}, t\right) & =\lim _{k \rightarrow \infty} \sup _{x \in(1 / 2 k, 1]} \frac{(2 x / k)-\left(1 / k^{2}\right)}{t+(2 x / k)-\left(1 / k^{2}\right)} \\
& =\lim _{k \rightarrow \infty} \frac{(2 / k)-\left(1 / k^{2}\right)}{t}=0 .
\end{aligned}
$$


That is, the sequence $\left(f_{k}\right)$ is uniformly statistically intuitionistic fuzzy convergent to $x^{2}$ on $[-1,1]$.

Remark 2.13. If $s t_{\mu, v}-f_{k} \rightrightarrows f$, then $s t_{\mu, v}-f_{k} \rightarrow f$. But the converse of this is not true.

We prove this with the following example.

Example 2.14. Let us define the sequence of functions

$$
f_{k}(x)= \begin{cases}0 & k=n^{2} \\ \frac{k^{2} x}{1+k^{3} x^{2}} & \text { otherwise }\end{cases}
$$

on $[0,1]$. This sequence of functions is pointwise statistically intuitionistic fuzzy convergent to 0 (indeed, for $x=1 / k, s t_{\mu, v}-f_{k}(1 / k)=1$ and for $\left.x=0, s t_{\mu, v}-f_{k}(0)=0\right)$. But, it is not uniformly statistical intuitionistic fuzzy convergent. Since the sequence of functions is bounded on $[0,1]$, we can use Proposition 2.10 to prove our claim. Let us take infumum for $\mu\left(f_{k}(x)-0, t\right)=\mu\left(k^{2} x /\left(1+k^{3} x^{2}\right), t\right)=t /\left(t+\left(k^{2} x\right) /\left(1+k^{3} x^{2}\right)\right)$ and supremum for $v\left(f_{k}(x)-\right.$ $0, t)=\left(v\left(k^{2} x /\left(1+k^{3} x^{2}\right), t\right)\right)=\left(k^{2} x /\left(1+k^{3} x^{2}\right) /\left(t+\left(k^{2} x\right) /\left(1+k^{3} x^{2}\right)\right)\right.$, over all $x \in[0,1]$. Firstly, we try to find $\sup _{x \in[0,1]}\left(k^{2} x /\left(1+k^{3} x^{2}\right)\right)$ and $\inf _{x \in[0,1]}\left(k^{2} x /\left(1+k^{3} x^{2}\right)\right)$. For this, we have

$$
\left(\frac{k^{2} x}{1+k^{3} x^{2}}\right)^{\prime}=\frac{k^{2}\left(1-k^{3} x^{2}\right)}{\left(1+n^{3} x^{2}\right)^{2}}=0 \Longrightarrow x=k^{-3 / 2}
$$

Since $f_{k}(0)=0, f_{k}(1)=k^{2} /\left(1+k^{3}\right)$ and $f_{k}\left(k^{-3 / 2}\right)=\sqrt{k} / 2$, we get $\sup _{x \in[0,1]}\left(k^{2} x /\left(1+k^{3} x^{2}\right)\right)=$ $\sqrt{k} / 2$ and $\inf _{x \in[0,1]}\left(k^{2} x /\left(1+k^{3} x^{2}\right)\right)=0$. Then,

$$
\begin{aligned}
\frac{k^{2} x}{1+k^{3} x^{2}} \leq \frac{\sqrt{k}}{2} & \Longrightarrow t+\frac{k^{2} x}{1+k^{3} x^{2}} \leq t+\frac{\sqrt{k}}{2} \\
& \Longrightarrow \frac{1}{t+(\sqrt{k} / 2)} \leq \frac{1}{t+\left(k^{2} x /\left(1+k^{3} x^{2}\right)\right)} \\
& \Longrightarrow \frac{t}{t+(\sqrt{k} / 2)} \leq \frac{t}{t+\left(k^{2} x /\left(1+k^{3} x^{2}\right)\right)}, \\
0 \leq \frac{k^{2} x}{1+k^{3} x^{2}} & \Longrightarrow t+0 \leq t+\frac{k^{2} x}{1+k^{3} x^{2}} \\
& \Longrightarrow \frac{1}{t+\left(k^{2} x /\left(1+k^{3} x^{2}\right)\right)} \leq \frac{1}{t} \\
& \Longrightarrow \frac{k^{2} x /\left(1+k^{3} x^{2}\right)}{t+\left(k^{2} x /\left(1+k^{3} x^{2}\right)\right)} \leq \frac{k^{2} x /\left(1+k^{3} x^{2}\right)}{t} \leq \frac{\sqrt{k}}{2 t}
\end{aligned}
$$


so, we get

$$
\begin{aligned}
\lim _{k \rightarrow \infty} \inf \mu\left(\frac{k^{2} x}{1+k^{3} x^{2}}, t\right) & =\lim _{k \rightarrow \infty} \inf \frac{t}{\left(t+k^{2} x /\left(1+k^{3} x^{2}\right)\right)}=\lim _{k \rightarrow \infty} \frac{t}{t+\sqrt{k} / 2} \\
& =\lim _{k \rightarrow \infty} \frac{2 t}{2 t+\sqrt{k}}=0 \neq 1 \\
\lim _{k \rightarrow \infty} \sup v\left(\frac{k^{2} x}{1+k^{3} x^{2}}, t\right) & =\lim _{k \rightarrow \infty} \sup \frac{k^{2} x /\left(1+k^{3} x^{2}\right)}{t+\left(k^{2} x /\left(1+k^{3} x^{2}\right)\right)}=\lim _{k \rightarrow \infty} \frac{\sqrt{k}}{2 t}=\infty \neq 0 .
\end{aligned}
$$

Therefore, we conclude that $\left(f_{k}\right)$ does not intuitionistic fuzzy uniformly statistical convergent to 0 .

Theorem 2.15. Let $f_{k}:(X, \mu, v, *, \diamond) \rightarrow\left(Y, \mu^{\prime}, v^{\prime}, *, \diamond\right)$ be a sequence of functions. If $\left(f_{k}\right)$ is uniformly intuitionistic fuzzy convergent on $X$ to a function $f$ with respect to $(\mu, v)$, then $s_{\mu, v}-f_{k} \rightrightarrows$ $f$. But the converse of this is not true.

Proof. Let $f_{k}$ be uniformly intuitionistic fuzzy convergent on $X$ to a function $f$. In this case, given $0<\varepsilon<1, t>0$, there exist a positive integer $k_{0}=k_{0}(r, t)$ such that $\forall x \in X$ and $\forall k>k_{0}$,

$$
\mu^{\prime}\left(f_{k}(x)-f(x), t\right)>1-\varepsilon, \quad v^{\prime}\left(f_{k}(x)-f(x), t\right)<\varepsilon .
$$

That is, for $k \leq k_{0}$

$$
\mu^{\prime}\left(f_{k}(x)-f(x), t\right) \leq 1-\varepsilon, \quad v^{\prime}\left(f_{k}(x)-f(x), t\right) \geq \varepsilon,
$$

is satisfied and these $k^{\prime}$ s are finite. Since finite set has 0-density, density of complement of finite set is 1 . If complement of this finite set is stated by $M$, for every $\varepsilon>0$, there exist $M \subset \mathbb{N}, \delta(M)=1$ and $\exists k_{0}=k_{0}(\varepsilon, t) \in M$ such that $\forall k>k_{0}$ and $k \in M$ and $\forall x \in X$,

$$
\mu^{\prime}\left(f_{k}(x)-f(x), t\right)>1-\varepsilon, \quad v^{\prime}\left(f_{k}(x)-f(x), t\right)<\varepsilon .
$$

This shows that $s t_{\mu, v}-f_{k} \rightrightarrows f$.

Definition 2.16. Let $f_{k}:(X, \mu, v, *, \diamond) \rightarrow\left(Y, \mu^{\prime}, v^{\prime}, *, \diamond\right)$ be a sequence of functions. The sequence $\left(f_{k}\right)$ is a uniformly statistically Cauchy sequence in intuitionistic fuzzy normed space provided that for every $\varepsilon>0$ and $t>0$, there exists a number $N=N(\varepsilon, t)$ such that

$$
\delta\left(\left\{k \in \mathbb{N}: \mu^{\prime}\left(f_{k}(x)-f_{N}(x), t\right) \leq 1-\varepsilon \text { or } v^{\prime}\left(f_{k}(x)-f_{N}(x), t\right) \geq \varepsilon \forall x \in X\right\}\right)=0 .
$$

Theorem 2.17. Let $f_{k}:(X, \mu, v, *, \diamond) \rightarrow\left(Y, \mu^{\prime}, v^{\prime}, *, \diamond\right)$ be sequence of functions. If $\left(f_{k}\right)$ is a uniformly statistically convergent sequence with respect to intuitionistic fuzzy norm $(\mu, v)$, then $\left(f_{k}\right)$ is uniformly statistically Cauchy sequence with respect to intuitionistic fuzzy norm $(\mu, v)$. 
Proof. Suppose that $s t_{\mu, v}-f_{k} \rightrightarrows f$. In this case, $\forall \varepsilon>0$, there exists $M \subset \mathbb{N}, \delta(M)=1$ and $k_{0}=k_{0}(\varepsilon, t) \in M$ such that $\forall k>k_{0}, k \in M$ and $\forall x \in X$,

$$
\mu^{\prime}\left(f_{k}(x)-f(x), \frac{t}{2}\right)>1-\varepsilon, \quad v^{\prime}\left(f_{k}(x)-f(x), \frac{t}{2}\right)<\varepsilon
$$

Choose $N=N(\varepsilon, t) \in M, N>k_{0}$. So, $\mu^{\prime}\left(f_{N}(x)-f(x), t / 2\right)>1-\varepsilon$ and $v^{\prime}\left(f_{N}(x)-f(x), t / 2\right)<\varepsilon$. We investigate $N=N(\varepsilon, t)$ such that

$$
\delta\left(\left\{k \in \mathbb{N}: \mu^{\prime}\left(f_{k}(x)-f_{N}(x), t\right) \leq 1-\varepsilon \text { or } v^{\prime}\left(f_{k}(x)-f_{N}(x), t\right) \geq \varepsilon \forall x \in X\right\}\right)=0,
$$

or

$$
\delta\left(K^{\prime}\right)=\delta\left(\left\{k \in \mathbb{N}: \mu^{\prime}\left(f_{k}(x)-f_{N}(x), t\right)>1-\varepsilon \text { or } v^{\prime}\left(f_{k}(x)-f_{N}(x), t\right)<\varepsilon \forall x \in X\right\}\right)=1 .
$$

For every $k \in M$, we have

$$
\begin{aligned}
\mu^{\prime}\left(f_{k}(x)-f_{N}(x), t\right) & =\mu^{\prime}\left(f_{k}(x)-f(x)+f(x)-f_{N}(x), t\right) \\
& \geq \mu^{\prime}\left(f_{k}(x)-f(x), \frac{t}{2}\right) * \mu^{\prime}\left(f(x)-f_{N}(x), \frac{t}{2}\right) \\
& >(1-\varepsilon) *(1-\varepsilon) \\
& =(1-\varepsilon), \\
v^{\prime}\left(f_{k}(x)-f_{N}(x), t\right) & =v^{\prime}\left(f_{k}(x)-f(x)+f(x)-f_{N}(x), t\right) \\
& \leq v^{\prime \prime}\left(f_{k}(x)-f(x), \frac{t}{2}\right) * v^{\prime}\left(f(x)-f_{N}(x), \frac{t}{2}\right) \\
& >\varepsilon \diamond \varepsilon \\
& =\varepsilon .
\end{aligned}
$$

Since $\delta(M)=1,\left(f_{k}\right)$ is a uniformly statistically Cauchy sequence in intuitionistic fuzzy normed space.

Theorem 2.18. Let $(X, \mu, v, *, \diamond)$ and $\left(Y, \mu^{\prime}, v^{\prime}, *, \diamond\right)$ be two IFNS and the mapping $f_{k}$ : $(X, \mu, v, *, \diamond) \rightarrow\left(Y, \mu^{\prime}, v^{\prime}, *, \diamond\right)$ be the intuitionistic fuzzy continuous on $X$ of sequence of functions. If $s t_{\mu, v}-f_{k} \rightrightarrows f$, the mapping $f: X \rightarrow Y$ is the intuitionistic fuzzy continuous on $X$.

Proof. Let $x_{0} \in X$ be an arbitrary point. By the intuitionistic fuzzy continuity of $f_{k}$ 's, for every $\varepsilon>0$ and $t>0$ there exists $\delta=\delta\left(x_{0}, \varepsilon, t / 3\right)>0$ such that

$$
\mu^{\prime}\left(f_{k}\left(x_{0}\right)-f_{k}(x), \frac{t}{3}\right)>1-\varepsilon, \quad v^{\prime}\left(f_{k}\left(x_{0}\right)-f_{k}(x), \frac{t}{3}\right)<\varepsilon,
$$


for every $k \in \mathbb{N}$ and all $x$ such that $\mu\left(x_{0}-x, t\right)>1-\delta$ and $v\left(x_{0}-x, t\right)<\delta$. Let $x \in B\left(x_{0}, \delta, t\right)$ be fixed $\left(B\left(x_{0}, \delta, t\right)\right.$ stands for an open ball in $(X, \mu, v, *, \diamond)$ with center $x_{0}$ and radius $\left.\delta\right)$. Since $s t_{\mu, v}-f_{k} \rightrightarrows f$ on $X$, for all $x \in X$, if we state, respectively, $A$ and $B$ by these sets

$$
\begin{gathered}
A=\left\{k \in \mathbb{N}: \mu^{\prime}\left(f_{k}(x)-f(x), \frac{t}{3}\right) \leq 1-\varepsilon \text { or } v^{\prime}\left(f_{k}(x)-f(x), \frac{t}{3}\right) \geq \varepsilon \forall x \in X\right\}, \\
B=\left\{k \in \mathbb{N}: \mu^{\prime}\left(f_{k}\left(x_{0}\right)-f\left(x_{0}\right), \frac{t}{3}\right) \leq 1-\varepsilon \text { or } v^{\prime}\left(f_{k}\left(x_{0}\right)-f\left(x_{0}\right), \frac{t}{3}\right) \geq \varepsilon \forall x \in X\right\}
\end{gathered}
$$

then, $\delta(A)=0$ and $\delta(B)=0$, hence $\delta(A \cup B)=0$ and $A \cup B$ is different from $\mathbb{N}$. Thus, there exists $m \in \mathbb{N}$ such that

$$
\begin{array}{cc}
\mu^{\prime}\left(f_{m}(x)-f(x), \frac{t}{3}\right)>1-\varepsilon, & v^{\prime}\left(f_{m}(x)-f(x), \frac{t}{3}\right)<\varepsilon \\
\mu^{\prime}\left(f_{m}\left(x_{0}\right)-f\left(x_{0}\right), \frac{t}{3}\right)>1-\varepsilon, & v^{\prime}\left(f_{m}\left(x_{0}\right)-f\left(x_{0}\right), \frac{t}{3}\right)<\varepsilon .
\end{array}
$$

Now, we will show that $f$ is intuitionistic fuzzy contunious at $x_{0}$. Using Definition 1.1, we have

$$
\begin{aligned}
\mu^{\prime}\left(f(x)-f\left(x_{0}\right), t\right) & =\mu^{\prime}\left(f(x)-f_{m}(x)+f_{m}(x)-f_{m}\left(x_{0}\right)+f_{m}\left(x_{0}\right)-f\left(x_{0}\right), t\right) \\
& \geq \mu^{\prime}\left(f(x)-f_{m}(x), \frac{t}{3}\right) * \mu^{\prime}\left(f_{m}(x)-f_{m}\left(x_{0}\right), \frac{t}{3}\right) * \mu^{\prime}\left(f_{m}\left(x_{0}\right)-f\left(x_{0}\right), \frac{t}{3}\right) \\
& >(1-\varepsilon) *(1-\varepsilon) *(1-\varepsilon) \\
& =1-\varepsilon, \\
v^{\prime}\left(f(x)-f\left(x_{0}\right), t\right) & =v^{\prime}\left(f(x)-f_{m}(x)+f_{m}(x)-f_{m}\left(x_{0}\right)+f_{m}\left(x_{0}\right)-f\left(x_{0}\right), t\right) \\
& \leq v^{\prime}\left(f(x)-f_{m}(x), \frac{t}{3}\right) * v^{\prime}\left(f_{m}(x)-f_{m}\left(x_{0}\right), \frac{t}{3}\right) * v^{\prime}\left(f_{m}\left(x_{0}\right)-f\left(x_{0}\right), \frac{t}{3}\right) \\
& <\varepsilon \diamond \varepsilon \diamond \varepsilon \\
& =\varepsilon .
\end{aligned}
$$

Thus, the proof is completed.

\section{Acknowledgments}

This work is supported by The Scientific and Technological Research Council of Turkey (TÜBITAK) under the Project number 110T699. We have benefited a lot from the report of the anonymous referee. So, the authors are thankful for his/her valuable comments and careful corrections on the first draft of this paper which improved the presentation and readability. 


\section{References}

[1] L. A. Zadeh, “Fuzzy sets," Information and Computation, vol. 8, pp. 338-353, 1965.

[2] L. C. Barros, R. C. Bassanezi, and P. A. Tonelli, "Fuzzy modelling in population dynamics," Ecological Modelling, vol. 128, no. 1, pp. 27-33, 2000.

[3] A. L. Fradkov and R. J. Evans, "Control of chaos: methods and applications in engineering," Annual Reviews in Control, vol. 29, no. 1, pp. 33-56, 2005.

[4] R. Giles, "A computer program for fuzzy reasoning," Fuzzy Sets and Systems, vol. 4, no. 3, pp. 221-234, 1980.

[5] L. Hong and J.-Q. Sun, "Bifurcations of fuzzy nonlinear dynamical systems," Communications in Nonlinear Science and Numerical Simulation, vol. 11, no. 1, pp. 1-12, 2006.

[6] J. Madore, "Fuzzy physics," Annals of Physics, vol. 219, no. 1, pp. 187-198, 1992.

[7] P. Das, "Fuzzy topology on fuzzy sets: product fuzzy topology and fuzzy topological groups," Fuzzy Sets and Systems, vol. 100, no. 1-3, pp. 367-372, 1998.

[8] R. Saadati and J. H. Park, "On the intuitionistic fuzzy topological spaces," Chaos, Solitons and Fractals, vol. 27, no. 2, pp. 331-344, 2006.

[9] K. T. Atanassov, "Intuitionistic fuzzy sets," Fuzzy Sets and Systems, vol. 20, no. 1, pp. 87-96, 1986.

[10] M. Mursaleen, S. A. Mohiuddine, and O. H. H. Edely, "On the ideal convergence of double sequences in intuitionistic fuzzy normed spaces," Computers $\mathcal{E}$ Mathematics with Applications, vol. 59, no. 2, pp. 603-611, 2010.

[11] M. Mursaleen, V. Karakaya, and S. A. Mohiuddine, "Schauder basis, separability, and approximation property in intuitionistic fuzzy normed space," Abstract and Applied Analysis, vol. 2010, Article ID 131868, 14 pages, 2010.

[12] Y. Y1lmaz, "Schauder bases and approximation property in fuzzy normed spaces," Computers $\mathcal{E}$ Mathematics with Applications, vol. 59, no. 6, pp. 1957-1964, 2010.

[13] J. H. Park, "Intuitionistic fuzzy metric spaces," Chaos, Solitons and Fractals, vol. 22, no. 5, pp. 1039-1046, 2004.

[14] A. George and P. Veeramani, "On some results in fuzzy metric spaces," Fuzzy Sets and Systems, vol. 64, no. 3, pp. 395-399, 1994.

[15] T. K. Samanta and I. H. Jebril, "Finite dimensional intuitionistic fuzzy normed linear space," International Journal of Open Problems in Computer Science and Mathematics, vol. 2, no. 4, pp. 574-591, 2009.

[16] B. Dinda and T. K. Samanta, "Intuitionistic fuzzy continuity and uniform convergence," International Journal of Open Problems in Computer Science and Mathematics, vol. 3, no. 1, pp. 8-26, 2010.

[17] H. Fast, "Sur la convergence statistique," vol. 2, pp. 241-244, 1951.

[18] M. Balcerzak, K. Dems, and A. Komisarski, "Statistical convergence and ideal convergence for sequences of functions," Journal of Mathematical Analysis and Applications, vol. 328, no. 1, pp. 715-729, 2007.

[19] A. Caserta, G. Di Maio, and L. D. R. Kočinac, "Statistical convergence in function spaces," Abstract and Applied Analysis, vol. 2011, Article ID 420419, 11 pages, 2011.

[20] A. Caserta and L. D. R. Kocinac, "On statistical exhaustiveness," Applied Mathematics Letters, vol. 25, no. 10, pp. 1447-1451, 2012.

[21] G. Di Maio and L. D. R. Kočinac, "Statistical convergence in topology," Topology and its Applications, vol. 156, no. 1, pp. 28-45, 2008.

[22] O. Duman and C. Orhan, " $\mu$-statistically convergent function sequences," Czechoslovak Mathematical Journal, vol. 54, no. 2, pp. 413-422, 2004.

[23] S. Karakus, K. Demirci, and O. Duman, "Statistical convergence on intuitionistic fuzzy normed spaces," Chaos, Solitons and Fractals, vol. 35, no. 4, pp. 763-769, 2008.

[24] B. Schweizer and A. Sklar, "Statistical metric spaces," Pacific Journal of Mathematics, vol. 10, pp. 313334, 1960.

[25] H. Efe and C. Alaca, "Compact and bounded sets in intuitionistic fuzzy metric spaces," Demonstratio Mathematica, vol. 40, no. 2, pp. 449-456, 2007.

[26] J. A. Fridy, “On statistical convergence," Analysis, vol. 5, no. 4, pp. 301-313, 1985. 


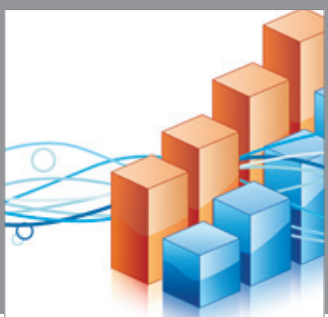

Advances in

Operations Research

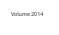

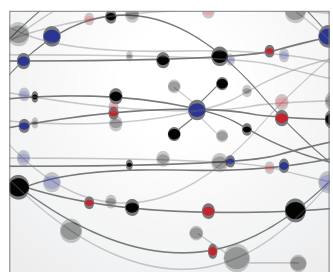

\section{The Scientific} World Journal
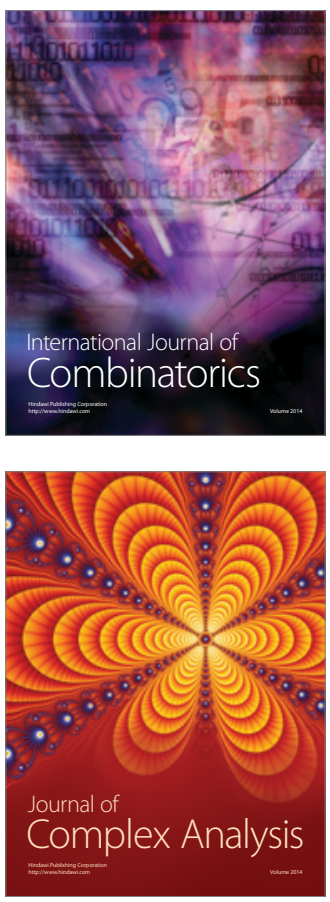

International Journal of

Mathematics and

Mathematical

Sciences
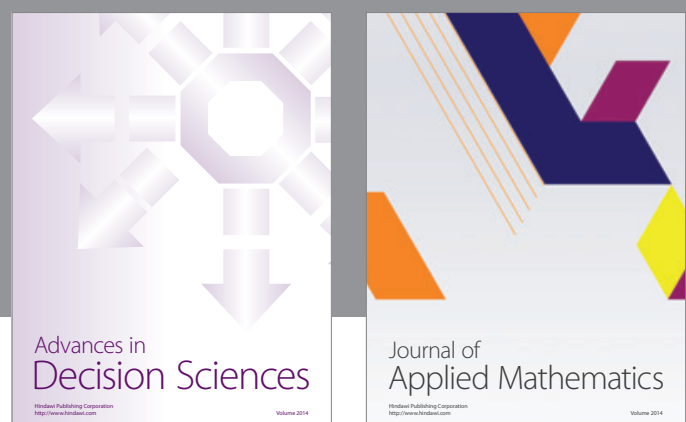

Journal of

Applied Mathematics
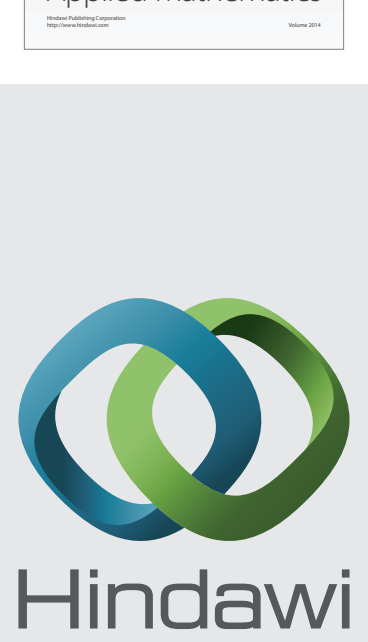

Submit your manuscripts at http://www.hindawi.com
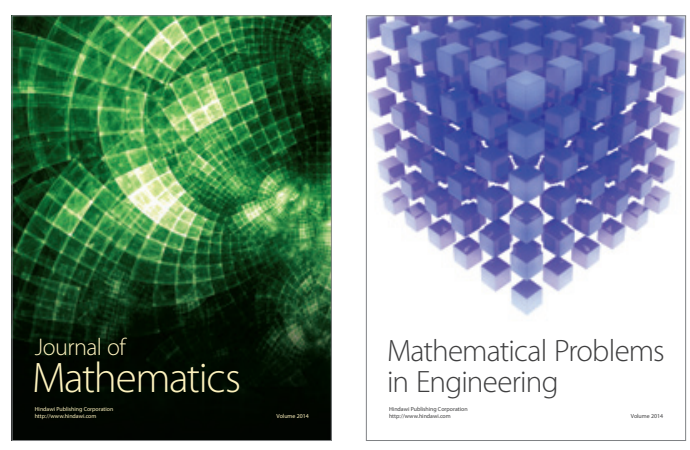

Mathematical Problems in Engineering
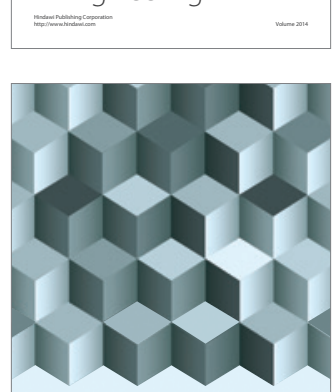

Journal of

Function Spaces
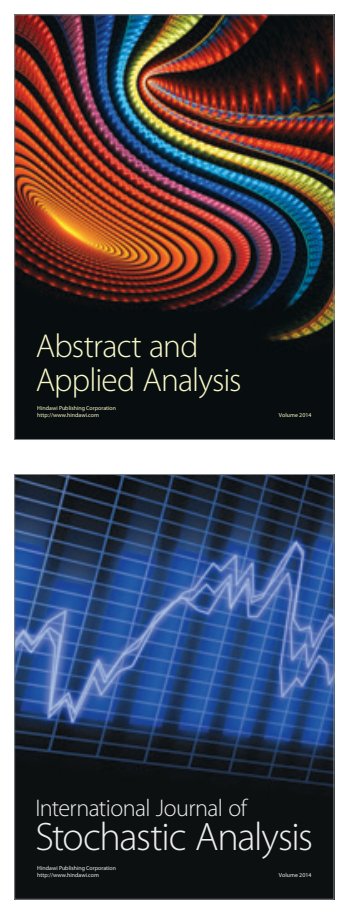

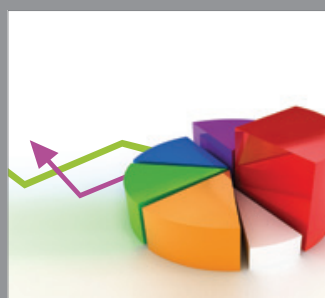

ournal of

Probability and Statistics

Promensencen
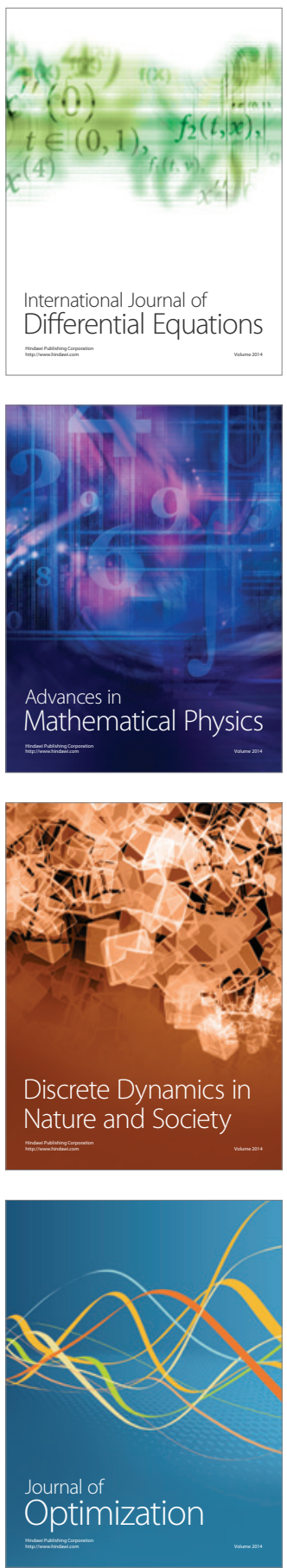